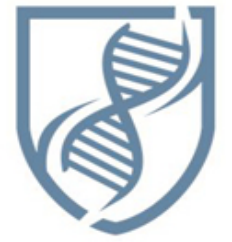

Journal of Bioscience and Applied Research
JBAAR

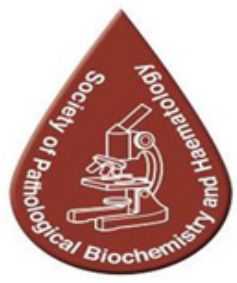

WWW.JBAAR.ORG

\title{
Comparative Study between Polymerase Chain Reaction and Different Rapid Tests for Detection of Mycobacterium Infection
}

\author{
Elarabany N. F ${ }^{1}$, Abdallah, A. E. ${ }^{1}$, Abdallah, G. A. ${ }^{2,}$ and Salah A. ${ }^{2}$. \\ ${ }^{1}$ Zoology Department, Faculty of Science, Damietta University \\ ${ }^{2}$ Microbiology and Immunology Department, Faculty of Medicine, Zagazig University, Egypt \\ *Corresponding author e.mail: elarabany@du.edu.eg
}

\begin{abstract}
The objective of this work is to reach a sensitive and rapid method for the laboratory diagnosis of active tuberculosis disease in patients and latent TB infection in household contacts and healthy community controls. We recruited 86 subjects divided into 3 groups: Thirty six patients with suspected active TB (Twenty four suspected pulmonary TB cased and 12 suspected extrapulmonary TB cases). 28 household contacts of confirmed pulmonary TB patients and 22 healthy community controls. Methods: For diagnosis of active tuberculosis the following tests were evaluated: ZiehlNeelsen (Z-N) staining and culture on LownesteinJensen (L-j), tuberculin skin test (TST), nested polymerase chain reaction (PCR) assay on separated peripheral blood mononuclear cells (PBMCs), antibody detection by Immunchromatographic test (ICT), and JFN $\gamma$ assay by QuantiFERON TB Gold In-Tube method for diagnosis of latent tuberculosic the following tests were evaluated: TST and Quantiferon TB Gold (In-tube method). The results showed that in pulmonary TB group, TST was the most sensitive test $(92.3 \%)$, while QFT-Gold IT plus ZN was the most sensitive combined test (100\%). In extrapulmonary TB group, TST, nested PCR and QFT-Gold IT were the most sensitive single tests (75\%) while QFT-Gold IT plus ZN was the most sensitive combines test (100\%). For diagnosis of LTBI: QFT-Gold IT was as sensitive as the TST. TST was 54.5\% specific, while QFT Gold-IT was 94.5\% specific TST showed that the exposure in the contacts group increased the risk of infection in the contacts group 0.9 times more than the control group (OR, 0,9m 95\% Cl, 0.25-3.22; $\mathrm{P}=0.85$ ). by $\mathrm{QFT}$-Gold IT, exposure
\end{abstract}

increased the risk of infection in the contacts group 8.4 times more than the control group (OR, 8.4;95\% Cl, 0.9-195.63; $\mathrm{P}=0.028)$. In household contacts group the concordance between TST and QFT-Gold IT was poor (64.3\%, with a $\mathrm{k}$ value of $0.23 \pm 0.18$ ). In conclusion, using QFT-Gold IT plus ZN staining is very helpful in the diagnosis of active TB disease. For diagnosis of LTBl. QuantiFERON-TB Gold In-Tube method is more helpful than Tuberculin skin test.

Key words. Tuberculosis, latent, TB, QFT-Gold IT, tuberculin.

\section{Introduction}

Tuberculosis (TB) is a major health problem all over the world. Definitive diagnosis of active tuberculosis usually involves laboratory isolation and identification of the infecting microorganism (Forbes et al., 2007). Conventional approaches to diagnosis continue to rely on tests that have major drawbacks as sputum smear microscopy, culture and tuberculin skin testing (TST). So there is a need for more rapid accurate tests (Harada et al., 2012). Various rapid diagnostic solutions appeared as nested polymerase chain reaction (PCR) (Hanore et al., 2008), Immunchromatographic test (ICT) (Steingart et al., 2013) and QuantiFERON $®$-TB Gold In-Tube (QFT-Gold IT) (Lalvani and Pareek, 2014).

Latent TB infection (LTBI) is an asymptomatic state with no clinical or radiological evidence of active disease but with living inactive $M$. tuberculosis organisms within tissues. Accurate diagnosis of latent tuberculosis infection is very difficult. Tuberculin skin test has been the primary tool for identifying latent TB infection. However QuantiFERON®-TB Gold In-Tube 
appeared as an alternative. It has many advantages over the TST in the diagnosis of LTBl (Lalvani and Pareek, 2014).

The objective of this work was to reach a sensitive and rapid method for the laboratory diagnosis of active mycobacterial tuberculosis infection in patients and Latent mycobacterial tuberculosis infection in household contacts and healthy community controls.

\section{Subjects and Methods}

This is a comparative cross sectional study conducted in the Microbiology and Immunology Department, Faculty of Medicine, Zagazig University. This study included 86 subjects distributed as follows:

Thirty six patients with suspected active TB were included in group I. it included 24 patients with suspected pulmonary TB, recruited from Zagazig Chest Hospital and the Chest Department in Zagazig University Hospitals and 12 patients with suspected extrapulmonary TB recruited from different departments in Zagazig University Hospitals. Patients were considered to be clinically suspected cases of TB if they had general symptoms typical of TB (asthenia, weight loss, anorexia prolonged fever resistant to broadspectrum antibiotics and night sweat). In addition, for pulmonary TB, respiratory symptoms (cough, dyspnea, or hemoptysis) for more than 2 weeks and radiographic findings consistent with TB were considered and for each extrapulmonary TB site, specific elements were considered.

Twenty eight household contacts of confirmed pulmonary TB patients were recruited group II.

Twenty two healthy community controls without history of exposure to TB patient were recruited in group III.

All subjects in group II and III had no clinical symptoms or radiographic signs suggestive of active tuberculosis. They had negative results for $\mathrm{ZN}$ staining and culture on L-J medium.

For diagnosis of active tuberculosis, all patients in group I were subjected to:

1.Ziehl-Neelsen (Z-N) staining and culture on Löwnestein (L-J) medium-for clinical specimens as described before (Forbes et al., 2007).

2.Tuberculin skin test (TST) according to guidelines of American Thoracic Society (American Thoracic Society, 2010).

3.Nested polymerase chain reaction (PCR) assay on separated peripheral blood mononuclear cells (PBMCs): -The PBMCs were separated by Ficoll-Hypaque centrifugation (Hanore et al., 2008).

-Extraction of DNA from the PBMCs by QIAamp DNA Mini Kit (Qiagen, Germany) was done following manufacturer's instructions.

-Two pairs of primers amplifying the gene sequence encoding the MPB64 protein of M. tuberculosis were prepared. outer forward primer sequence $5 `$-ATC CGC TGC CAG TCG TCT TCC-3`, outer reverse primer sequence: 5`CTC GVG AGT CTA GGC CAG CAT-
3`, inner forward primer sequence: 5 -CAT TGT GCA AGG TGA ACT GAG C-3` and inner reverse primer sequence: 5`-AGC ATC GAG TCG ATC GCG GAA-3`.

The reaction mixture consisted of $10 \mathrm{pmol}(2 \mu \mathrm{l})$ of each primer, $14 \mu$ l of the master mix (Tag PCR Master Mix Kit (Qiagen, GmbH, Germany)). Then $2 \mu \mathrm{l}$ of the product of the extraction was added to the Master Mix in each tube. Thus the total volume of the PCR reaction mixture was in $20 \mu$ l (Takahashi and Nakayama, 2011). The samples were amplified for 35 cycles each cycle consisted of denaturation at $95.0^{\circ} \mathrm{C}$ for 30 seconds, annealing at $60^{\circ} \mathrm{C}$ for 30 seconds and primer extension at $72^{\circ} \mathrm{C}$ for I minute. For nested PCR: It was carried out for 25 cycles at $94^{\circ} \mathrm{C}$ for 30 seconds, $55^{\circ} \mathrm{C}$ for 30 seconds and $72^{\circ} \mathrm{C}$ for 1 minute. Before both single step PCR and nested PCR, initial denaturation was carried out for one cycle at $96.0^{\circ} \mathrm{C}$ for 3 minutes. After both single step PCR and nested PCR, final extension was carried out for one cycle at $72^{\circ} \mathrm{C}$ for 1 minute. Positive and negative controls were included in each run, and all precautions to prevent cross-contamination were observed. Amplified product was visualized after electrophoresis in a $2 \%$ agarose gel in Tris-borateEDTA buffer. Target bands of approximately 239 and 194 bp were visualized by staining with ethidium bromide (Takahashi and Nakayama, 2011).

For the presence of PCR inhibitors we included internal amplification control for samples which gave negative results. We amplified a synthetic target gene (The base sequence of target gene as the information was divulged by the manufacturer) with its hydrolysis probe. The new reaction carried out by real time PCR (Light cycler 480 Roche) under the following conditions: one cycle of 10 minutes at $95^{\circ} \mathrm{C}$ followed by 45 cycle ( 2 step cycle consisted of $95^{\circ} \mathrm{C}$ for 10 seconds. $95^{\circ} \mathrm{C}$ for 10 seconds). Then one cycle of 1 minute at $40^{\circ} \mathrm{C}$.

4.Antibody detection by Immunchromatographic test (ICT): It was done by TB Rapid Test Device (Acon Labo Inc). There drops of anticoagulated blood about $(75 \mu \mathrm{l})$ were transferred to the specimen well of test device. One drop of buffer (approximately 40 $\mu$ ) was added. The result was read after 10-30 minutes. Positive: if two distinct colored lines appeared. One line appeared in the control region and another line in the test region. Negative: if one coloured line appeared in the control region. Invalid: if control line failed to appear.

5.Interferon-Gamma assay (IFN $\gamma$ ) assay by Quantiferon TB Gold In-tube method (QFT-Gold IT): Manufacture instructions were followed precisely. QuantiFERON-TB Gold In-Tube (Cellestis Ltd. Carnegie, Australia) was supplied with three heparinized blood collection tubes. First tube is precoated with three TB-specific antigens (ESAT-6, CFP-10, and TB7.7). Second tube is precoated with a mitogen (phytohemagglutinin), and a third tube coated with anticoagulant only (Negative control tube). Three milliliters of blood ( $1 \mathrm{ml}$ for each tube) was 
collected prior to TST administration. The tubes were mixed by shaking vigorously for 5 to 10 seconds to ensure that all surfaces of the tube were coated with blood and that complete mixing of the contents bad occurred. The tubes were incubated upright overnight (16 to $24 \mathrm{~h}$ ) at $37^{\circ} \mathrm{C}$ and then centrifuged at $4000 \mathrm{rpm}$ for $10 \mathrm{~min}$. plasma was harvested from each tube. Plasma samples were tested by an enzyme-linked immunosorbent assay (ELISA), whose IFN- $\gamma$ limit of detection is $0.05 \mathrm{IU} / \mathrm{ml}$. The Optical Density (OD) was measured using a microplate reader fitted with a $450 \mathrm{~nm}$ filter and with a 620nm to 650nm reference filter. QuantiFERON TB Gold Analysis software was used to calculate results from the raw optical densities. The manufacture's cut off a positive teat, indicating M. tuberculosis infection, is $0.35 \mathrm{IU} / \mathrm{ml}$ of IFN- $\gamma$ for the TB-specific antigen-stimulated plasma sample above the amount of IFN- $\gamma$ in the negative control sample (Cellestis, 2012).

For diagnosis of latent tuberculosis, all subjects in group II and III were subjected to TST (American Thoracic Society, 2010) and QFT-Gold IT (Cellestis, 2012).

There is no "gold standard" reference test for diagnosis of latent TB infection. Instead the following parameters were measured: 1 . Sensitivity of the test from active pulmonary TB cases. 2. Specificity of the test from controls. 3. The correlation of each test to exposure as a risk of latent TB infection (Lalvani and Pareek, 2014).

Statistical analysis: Data were coded, entered and analysed using SPSS version 15.

\section{Results}

\section{Results of diagnostic tests among suspected tuberculous patients:}

Culture on L-J was considered the gold standard test. In suspected pulmonary TB patients, cultivation of 24 sputum samples revealed $13(54.2 \%)$ positive cases (9 males, 4 females, age range 16-45 years). In suspected extrapulmonary TB patients, cultivation of 12 clinical samples revealed 4 (33.3\%) positive cases (3 males, one female, age range 18-55 years). In pulmonary TB group, TST was the most sensitive test $(92.3 \%)$ and $\mathrm{ZN}$ was the most specific one (90.9\%). In extrapulmonary TB group. TST nested PCR and QFT-Gold IT were the most sensitive tests (75\%) and $\mathrm{ZN}$ was the most specific (100\%) test (Table1).

We examined the validity of combined QFT Gold IT with other tests. In pulmonary TB group. QFT-Gold IT plus $\mathrm{ZN}$ was the most sensitive combined test (100\%). In extrapulmonary TB group TST, nested PCR and QFTGold IT were the most sensitive single tests (75\%) while GFT-Gold IT plus ZN was the most sensitive combined test (100\%) (Table2).

Results of diagnostic teats for latent TB among contacts and controls:

In group II (Household contacts), positive TST result were obtained in 12 out of 28 subjects (42.9\%) [5 males,
7 females, age range 17-51 years]. For IFN- $\gamma$ determination by QFT-Gold IT assay there were 8 out of $28(28.6 \%)$ positive results [3 males, 5 females, age range 17-55 years]. In group III (control group), positive TST results were obtained in 10 out of 22 (45.5\%) [4 males, 6 females, age range 18-55 tears]. For IFN- $\gamma$ determination by QFT-Gold IT assay there were only one subject out of 22 was positive by QFT Gold-IT (4.65\%) [ A 33 years old female], 21 negative cases out of 22 (95.45\%). The specificity of TST in control group was $54.5 \%$.

The specificity of QFT-Gold IT in control group was 94.5\%. The correlation of each test to exposure was measured by estimating the odds ratio (OR). TST showed that the exposure in the contacts group increased the risk of infection 0.9 times more than the control group (OR, 0.9, 95\% Cl, 0.25-3.22, $\mathrm{P}=0.85$ ). By QFT-Gold IT, exposure increased the risk of infection in the contacts group 8.4 times more than the control group (OR, 8.4, 95\% Cl, 0.9-195.63: $\mathrm{P}=0.028$ )(Table 3).

The agreement between test results from the TST and the IFN- $\gamma$ assay was assessed using Kappa coefficients. In group II (Household contacts) the concordance between TST and QFT-Gold IT was poor (64.3\% with a k value of $0.23 \pm 0.18$ ). In group III (Healthy community controls), the agreement between TST and QFT-Gold IT was poor (95\% with a $\mathrm{k}$ value of $0.23 \pm 0.18$ ) (Table 4).

\section{Discussion}

In order to improve TB diagnosis, rapid and reliable tests are needed. So in the present study, we aimed to reach a sensitive and rapid method for the laboratory diagnosis of: active tuberculosis disease and latent tuberculosis infection. This study included 86 subjects distributed as follows. Thirty six patients with suspected active TB (24 patients with suspected pulmonary TB and 12 patients with suspected extrapulmonary TB), 28 household contacts of confirmed pulmonary TB patients and 22 healthy community controls. For diagnosis of active tuberculosis, all patients in group I were subjected to: Ziehl-Neelsen (Z-N) staining and culture on Löwnestein-Jensen (L-J), tuberculin skin (TST), nested polymerase chain reaction (PCR) assay on separated peripheral blood mononuclear cells (PBMCs). IFN $\gamma$ assay by Quantiferon TB Gold In-tube method and antibody detection by Immunchromatographic test (ICT). For diagnosis of latent tuberculosis, all subjects in group II and III were subjected to tuberculin skin test and IFN $\gamma$ assay by Quantiferon TB Gold (In-tube method).

Diagnosis of active tuberculosis: In suspected pulmonary TB patients, ZN achieved $76.9 \%$ sensitivity. Previous work in Sharkeia Governorate showed similar results as in the study of Asaad (2009) (76.9\%) and lower results as in the study of Marei et al. (2013) (54.5\%). This difference in sensitivity may be due to different decontamination techniques, different personal expertise (American Thoracic Society, 2010) and different bacillary load in the specimen (Hanore et al., 2008). 
Table (1): Validity of single rapid tests among suspected TB patients:

\begin{tabular}{llllllll}
\hline Studied Items & $+/$ total & Sensitivity & Specificity & PPV & NPV & Accuracy & P $^{*}$ \\
\hline $\begin{array}{l}\text { Pulmonary TB } \\
\text { - Z-N staining }\end{array}$ & $11 / 24$ & $76.9 \%$ & $90.9 \%$ & $90.9 \%$ & $76.9 \%$ & $83.3 \%$ & 0.0004 \\
\hline$\bullet \quad$ TST & $19 / 24$ & $92.3 \%$ & $36.4 \%$ & $63.2 \%$ & $80 \%$ & $66.7 \%$ & 0.042 \\
\hline$\bullet \quad$ Nested PCR & $13 / 24$ & $84.6 \%$ & $81.8 \%$ & $84.6 \%$ & $81.8 \%$ & $84.3 \%$ & 0.001 \\
\hline$\bullet \quad$ ICT & $10 / 24$ & $44.4 \%$ & $45.5 \%$ & $40 \%$ & $35.7 \%$ & $37.5 \%$ & 0.88 \\
\hline$\bullet \quad$ QFT Gold IT & $14 / 24$ & $84.6 \%$ & $72.7 \%$ & $78.6 \%$ & $80 \%$ & $79.2 \%$ & 0.002 \\
\hline $\begin{array}{l}\text { Extrapulmonary TB } \\
-\quad \text { ZN }\end{array}$ & $2 / 12$ & $59 \%$ & $100 \%$ & $100 \%$ & $80 \%$ & $83.3 \%$ & 0.014 \\
\hline$\bullet \quad$ TST & $7 / 12$ & $75 \%$ & $50 \%$ & $42.9 \%$ & $80 \%$ & $58.3 \%$ & 0.2 \\
\hline$\bullet \quad$ Nested PCR & $4 / 12$ & $75 \%$ & $62.5 \%$ & $25 \%$ & $62.5 \%$ & $50 \%$ & 0.66 \\
\hline$\bullet \quad$ QFT Gold IT & $4 / 8 * *$ & $75 \%$ & $75 \%$ & $75 \%$ & $75 \%$ & $75 \%$ & 0.07 \\
\hline
\end{tabular}

* $\mathrm{P}$ value $\leq 0.05$ is statistically significant.

** Four cases in the extrapulmonary TB group had indeterminate results so they were excluded from further analysis.

Table (2): Results of combined QFT Gold IT with other tests among suspected TB patients:

\begin{tabular}{llllllll}
\hline Studied Items & $+/$ total & Sensitivity & Specificity & PPV & NPV & Accuracy & P \\
\hline $\begin{array}{l}\text { Pulmonary TB } \\
\text { - QFT Gold IT+TST }\end{array}$ & $20 / 24$ & $92.3 \%$ & $27.3 \%$ & $60 \%$ & $75 \%$ & $62.3 \%$ & 0.09 \\
\hline$\bullet \quad$ QFT Gold IT+ZN & $16 / 24$ & $100 \%$ & $72.7 \%$ & $81.3 \%$ & $100 \%$ & $87.5 \%$ & 0.001 \\
\hline$\bullet \quad$ QFT Gold IT+ICT & $19 / 24$ & $84.6 \%$ & $27.3 \%$ & $57.3 \%$ & $60 \%$ & $58.3 \%$ & 0.23 \\
\hline $\begin{array}{l}\text { Extrapulmonary TB } \\
\text { - GFT Gold IT+TST }\end{array}$ & $7 / 8^{* *}$ & $100 \%$ & $25 \%$ & $57.1 \%$ & $100 \%$ & $62.5 \%$ & 0.05 \\
\hline - GFT Gold IT+ZN & $5 / 8^{* *}$ & $100 \%$ & $75 \%$ & $80 \%$ & $100 \%$ & $87.5 \%$ & 0.014 \\
\hline$\bullet \quad$ GFT Gold IT+ICT & $4 / 8^{* *}$ & $75 \%$ & $75 \%$ & $75 \%$ & $75 \%$ & $75 \%$ & 0.07 \\
\hline
\end{tabular}

* $\mathrm{P}$ value $\leq 0.05$ is statistically significant.

** Four cases in the extrapulmonary TB group had indeterminate results so they were excluded from further analysis.

Table (3). Risk assessment in contacts group:

\begin{tabular}{llll}
\hline Studied items & Control (group III) & Contacts group (Group II) & P \\
\hline TST & 1 & $0.9(0.25-3.22)$ & 0.85 \\
OR (95\% Cl) & 1 & & \\
\hline QFT-Gold IT & 1 & $8.4(0.9-195.63)$ & 0.028 \\
Or (95\% Cl) & & \\
\hline
\end{tabular}

* $\mathrm{P}$ Value $\leq 0.05$ is statistically significant.

Table (4). Agreement between TST and QFT-Gold IT results:

\begin{tabular}{lllll}
\hline \multirow{2}{*}{ Studied Items } & \multicolumn{4}{c}{ TST \& QFT-Gold IT results } \\
\cline { 2 - 5 } & Agreement (\%) & Kappa & Rating ** & P value \\
\hline Group II (Household contacts) & $64.3 \%$ & $0.23 \pm 0.18$ & Poor & $0.08^{*}$ \\
\hline Group III (Healthy community controls) & $59 \%$ & $0.1 \pm 0.09$ & Poor & $0.13^{*}$ \\
\hline$*$ P value $\leq 0.05$ is statistically significant. & & & \\
$* * 0.75$, excellent agreement; 0.4 , poor agreement; and between 0.4 and 0.75 , fair to good agreement.
\end{tabular}




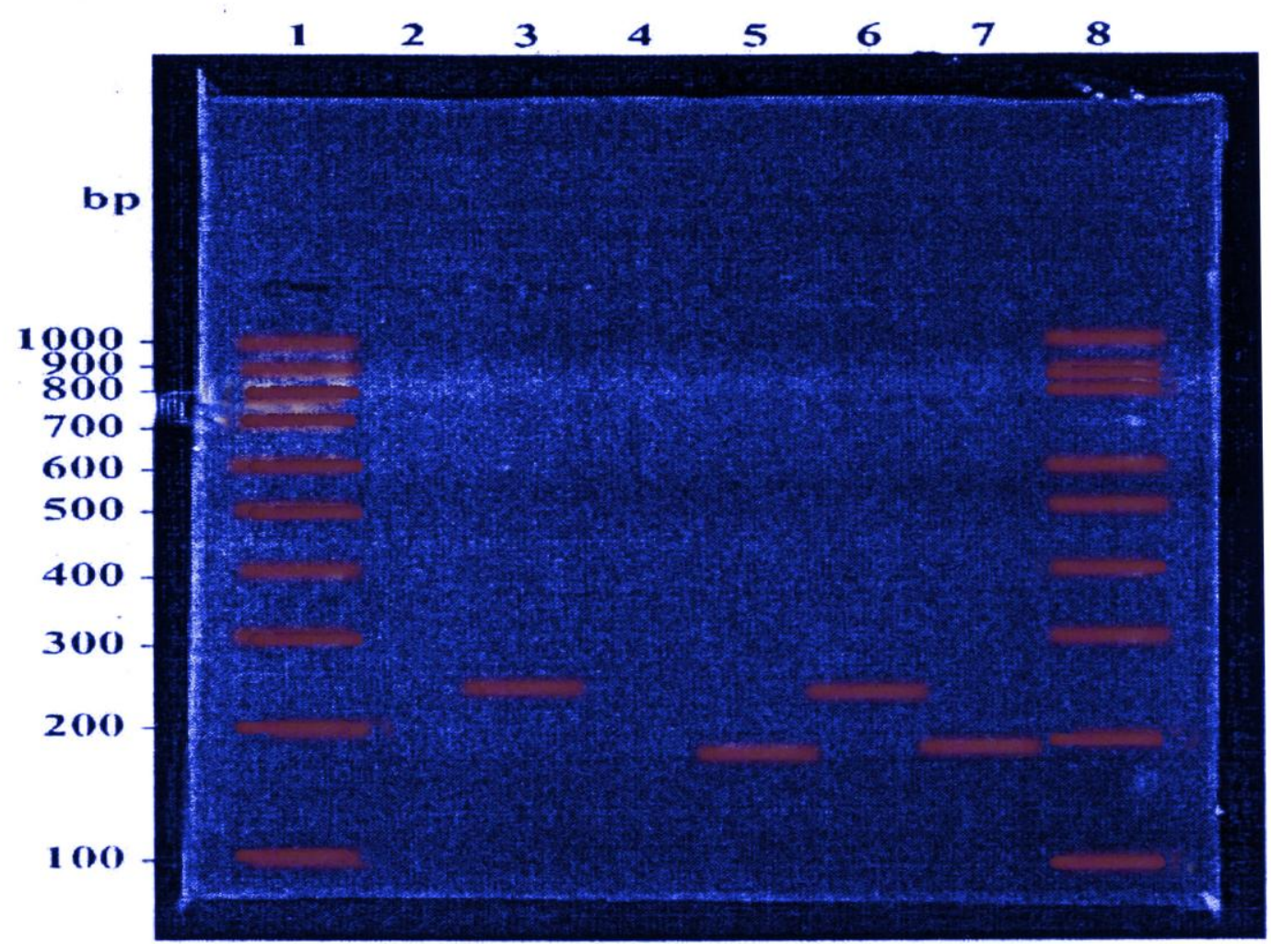

Figure (1). Electrophoresis analysis of amplified products of PCR assays

Lane 1,8: 100-bp DNA ladder, Lane 2: Negative control containing distilled water.

Lane 3,5: Sample positive for 239bp and 194 segments in MPB64 gene of M. tuberculosis

Lane 6,7: Positive control from culture confirmed strain, positive for 239 bp and 194 bp segments in MPB64 gene of M. tuberculosis. Lane 4: Negative control (Blank, reagents only).

In extrapulmonary $\mathrm{TB}$ patients group, $\mathrm{ZN}$ staining achieved 50\% sensitivity, which agreed with the American study of Torrea et al., (2014). Our results showed $100 \%$ specificity as in the Egyptian study of Amr (2010) (100\%).

When calculating the sensitivity of TST in suspected pulmonary TB group, it was $92.3 \%$. Our results agreed with Asaad (2009) (96.9\%), and Tsiouris et al. (2012) (90\%). Yet they were higher than those of Kang et al. (2014) from Korea (78\%). In suspected extrapulmonary TB patients, TST sensitivity was $75 \%$ versus $69 \%$ in the Korean study of Song et al. (2011). False negative TST results decrease test sensitivity. There may be biologic factors as use of immunosuppressant's and extremes of age or technical factors as improper storage and errors in administration. Technical factors are largely avoidable, although the biologic factors are not (AiJahdali et al., 2014). In our study we tried to avoid those technical defects. This was reflected on decrease false negative results in our work. In Pulmonary TB group, specificity for TST was 36.4\%. Different results were obtained from previous studies: (49\%) in the study of Kang et al. (2014) and (62.4\%) in the study of Asaad (2009). The discrepancy may occur as TST can't discriminate between active TB, latent TB, BCG vaccination and infection by other mycobacteria (AiJahdali et al., 2014). In extrapulmonary TB group, the specificity for TST was $50 \%$ which was near that of Song et al. (2011) who reported 57\% specificity.

In this study, PCR results revealed that among pulmonary TB patients, the sensitivity of nested PCR was $81.8 \%$. For extrapulmonary TB, the sensitivity was $75 \%$ by nested PCR. This goes with Martins et al. (2010) (Sensitivity was 70\% by nested PCR).

In this work, ICT achieved sensitivity $25 \%$ in suspected extrapulmonary TB and $30.8 \%$ in suspected pulmonary $\mathrm{TB}$. In pulmonary $\mathrm{TB}$, our results were similar to Ongut et al. (2013) who reported 33.3\% sensitivity. In extrapulmonary $\mathrm{TB}$, the results were much lower than the results of Rasolofo et al. (2011) who reported $65.2 \%$ sensitivity. Different sensitivity levels between previously mentioned studies may be because the antibody repertoire in TB is highly diversed (Lyashchenko et al., 2012).

Regarding the specificity of the QFT-Gold IT, it showed $72.7 \%$ specific in suspected pulmonary TB patients and $75 \%$ in suspected extrapulmonary TB. Our 
results were lower than Detjen et al. (2013) who reported 93\% specificity but higher than Tsiouris et al. (2012) who reported 65\% specificity. False positive results may led to decrease specificity in previously mentioned studies. False positive QFT-Gold IT results may be due to inclusion of some cases with LTBI (Nishimura et al., 2014). As QFT-Gold IT is promising for LTBI diagnosis so a positive QFT-Gold IT result means that there is TB infection. It cannot distinguish between LTBI and active TB disease i.e., a positive QFT-Gold IT result indicates either LTBI or active TB disease (Lalvani and Pareek, 2014).

For QFT-Gold IT to be useful in confirming active disease, it must be highly sensitive (Lalvani and Pareek, 2014). In suspected pulmonary TB, QFT-Gold IT had $84.6 \%$ sensitivity. Our results agreed with Kang et al. (2014) who recorded $81 \%$ sensitivity. For suspected extrapulmonary TB patients, the sensitivity of QFTGold IT was 75\%. Many studies were made on extrapulmonary TB. Their results showed sensitivities ranging from $14 \%$ to $80 \%$. This difference may be related to the diverse clinical presentations of extrapulmonary TB (Song et al., 2011). As sensitivity of QFT-Gold IT was suboptimum, so we examined the combined sensitivity of QFT-Gold IT with the TST or with $\mathrm{ZN}$ staining. In this regard, we found that in suspected pulmonary TB patients, the sensitivity of QFT-Gold IT plus TST was 92.3\%. It exceeded that of QFT-Gold IT alone (84.6\%). Our findings agreed with those of Tsiouris et al. (2012) (75\% was raised to 86\%). In the same group of patients, the sensitivity of QFTGold IT plus ZN was $100 \%$. This was consistent with the results of Raven et al. (2010) from Denmark (83\% was raised to 97\%). In suspected extrapulmonary TB group, the sensitivity of QFT-Gold IT was 75\%. It was raised to $100 \%$ by QFT-Gold IT plus ZN and $100 \%$ by QFT-Gold IT plus TST.

As both ICT and QFT-Gold IT are rapid tests and require only very small volume of blood so the role of combined ICT and QFT-Gold IT tests was evaluated. In this regards we found that although the sensitivity of combined tests is better than ICT alone (84.6\% sensitivity in pulmonary TB and $75 \%$ in extrapulmonary), however it didn't differ from the results of QFT-Gold IT alone (84\% sensitivity in pulmonary $\mathrm{TB}$ and $75 \%$ in extrapulmonary).

For QFT-Gold IT to exclude active TB it should have a high negative predictive value (Lalvani and Pareek, 2014). In this study, negative predictive value of QFT-Gold IT alone was 80\% in suspected pulmonary TB and $75 \%$ in suspected extrapulmonary TB. By using QFT-Gold IT combined with ZN we obtained a very negative predictive value (100\%) in suspected pulmonary and extrapulmonary TB which could allows for rapid exclusion of TB suspects from further investigations. Thus the combined strategy of using results of QFT-Gold IT plus ZN staining should be very helpful in diagnosis of active TB disease. Positive results in both could be used as an indicator for active TB disease, and negative results could exclude the diagnosis of active TB, this conclusion agreed with Raven et al. (2013) and Tsiouris et al. (2012).

Diagnosis of latent tuberculosis: accurate diagnosis of latent tuberculosis infection is very difficult. The tuberculin skin test has been the primary tool for identifying latent TB infection. Although the TST has been used in clinical practice, it has several major limitations. However, advances in the molecular biology of M. tuberculosis and modern immunological methods have led to the development of QFT-Gold IT. It has a number of potential advantages over the TST. They have excellent specificity. The test results are not affected by BCG vaccination of NTM infection. Other benefits are that they require only a single visit by the patient and pose no risk of serious skin reactions (Lalvani and Pareek, 2014).

In household contacts group (Group II), this study showed that positive TST results were obtained in 12/28 (42.9\%) of the subjects. Similar results were found in the Gambian study of Adetifa et al. (2013) (48.6\%). QFT-Gold IT results showed 8/28 (28.6\%) positive cases. This agreed with Hill et al. (2012) from Gambia (28\%).

In the absence of a gold standard, the absolute sensitivity of the QFT-Gold IT and the TST for diagnosing LTBI still remains unclear. So it is very difficult to demonstrate that any test is superior to TST. However studies attempting to quantify the performance of TST and QFT-Gold IT have therefore used surrogate markers for LTBI including: (1) Study test sensitivity in active pulmonary $\mathrm{TB}$ disease as a surrogate for LTBI. (2) Assess specificity in healthy controls. (3) Measure the level of contact with infections cases (Lalvani and Pareek, 2014).

The specificity of TST and QFT-Gold IT in latent TB infection was studied in healthy controls. For TST, it was $54.4 \%$ specific, while QFT-Gold IT was $94.5 \%$ specific. Similar results were obtained in previous studies: TST had 49\% specificity and QFT-Gold had 96\% specificity (Kang et al., 2014). To measure the level of contact with infections cases, we calculated the correlation of each test results with the degree of exposure to a source patient (Lalvani and Pareek, 2014). In our study QFT-Gold IT results were more closely associated with the risk of infection than the TST $(\mathrm{P}<0.01)$, this agreed with Kang et al. (2014).

\section{Conclusion}

1- $\quad$ ZN staining may be still recommended as it is a cheap rapid method. But negative results don't exclude 
TB and false positive results may occur with environmental mycobacteria.

2- $\quad$ Nested PCR assay on PBMCs may be a useful tool for diagnosis of active TB, especially if it is difficult to obtain a clinical specimen or in case of low bacillary load in the specimen.

3- $\quad$ The ICT is rapid and easy to perform without requiring special equipment. However, the sensitivity demonstrated by the test (Either single or combined with QFT-Gold IT) does not suggest, for the moment, that the test should replace the standard methods for the diagnosis of active tuberculosis.

4- QFT-Gold IT alone cannot distinguish between LTBI and active TB disease i.e. a positive QFT-Gold IT result indicates either LTBI or active TB disease. However the results of QFT-Gold IT plus ZN staining could be very helpful in diagnosis of active TB disease; positive results in both to indicate for active TB disease and negative results to exclude active TB.

For diagnosis of LTBI, QFT-Gold IT is as sensitive as and more specific than TST (As it tests for T-cell response to specific mycobacterial antigens so not affected by BCG vaccine or environmental mycobacteria). It correlates better with exposure than the TST. So QuantiFERON TB Gold In-Tube method will be more helpful than Tuberculin skin test for diagnosis of latent tuberculosis infection in household contacts and community controls.

\section{References}

Adetifa IM, Lugos MD. Hammond A, Jeffries D, Donkor S, Adegbola RA and Hill PC (2013). Comparison of two interferon gamma release assays in the diagnosis of Mycobacterium tuberculosis infection and disease in The Gambia. BMC Infectious Disease; 7:122-133.

Ai-Jahdali H, Baharoon S, Abba A, Memish Z, Alrajhi A, Albarrak A, Haddad Q, Hajjaj M, Pai M and Menzies D (2014). Saudi guidelines for testing and treatment of latent tuberculosis infection. Ann Saudi Med: 30(I): 38-49.

American Thoracic Society (2010). Targeted tuberculin testing and treatment of latent tuberculosis infection Am j Respir Crit Care Med; 161:S221-S247.

Amr G (2010). Value of modern microbiological techniques in the diagnosis of mycobacteria in different clinical samples. Thesis for MD degree in Clinical pathology, Zagazig University.

Assad A (2009). Polymerase chain reaction for detecting rifampicin-resistant M.tuberculosis. Thesis for MD degree in Microbiology and Immunology. Zagazig University.

Cellestis (2012). Quantiferon In-Tube Package Insert Cellestis [Online]. Available
Detjen AK, Keil T, Roll S, hauer B, Mauch H, Wahn U, Magdorf K (2013). Interferon-gamma release assays improve the diagnosis of tuberculosis and nontuberculous mycobacterial disease in children in a country with a low incidence of tuberculosis. Clin Infect Dis;45L 322-8.

Forbes BA, Sahm Df and Weissfeld AS (2007). Mycobacteria and other bacteria with unusual growth requirements In: Baily and Scott's diagnosis microbiology, $12^{\text {th }}$ ed Mosby Elsevier INC China; Ch, 45, pp: 875-509.

Hanore s, Vincensini JP, Hocqueloux L, Noguera ME, Farge D, Lagrage P and Herrmann JL (2008). Diagnostic value of a nested polymerase chain reaction assay on peripheral blood mononuclear cells from patients with pulmonary and extrapulmonary tuberculosis. Int J Tuberc Lung Dis; 5: 754-762.

Harada N, Higuchi K and Yoshiyama T (2012). Comparison of sensitivity and specificity of two blood interferon gamma assays for $\mathrm{M}$. tuberculosis infection, $\mathrm{J}$ Infect; 56: 348-353.

Hill PC, Brookes RH, Fox A, Jackson-Sillah D, Lugos MD, Jeggries DJ, Donkor SA, Adegbola R and McAdam K(2012). Surprisingly high specificity of the PPD skin test for $M$ tuberculosis infection from recent exposure in The Gambia. PloS One: l(I):e68.

http://www.cellestis.com/IRM/Company/ShPage.a spx?(PID-1171.|November 12.2008].

Kang YA, Lee HW, Yoon HI, Cho B, Han SK, Shim YS and Yim JJ (2014). Discrepancy between the tuberculin skin test and the whole-blood interferon gamma assay for the diagnosis of latent tuberculosis infection in an intermediate tuberculosis-burden. JAMA: 293: 2756-2761.

Lalvani A and Pareek M (2014). Interferon gamma relese assays: principles and practice. Enferm Infecc Microbial Clin: 28(4): 245-252.

Lyashchenko KP, Singh M, Colangeli R, and Gennaro M.L (2012). A multiantigen print immunoassay for the development of serological diagnosis of infectious diseases. J. Immunol Methods; 242: 91-100.

Marei AM, El-Behedy EN, Mohtad, HA and Afify AF (2013). Evaluation of a rapid bacteriophage-based method for the detection of Mycobacterium tuberculosis in clinical samples. J Medical Microbiology 52:331335.

Martins LC, Baschoal IA, Von Nowakonski A, Silva SAB, Costa FF and Ward LS (2010). Nested-PCR asing MPB64 fragment improves the diagnosis of pleural and meningeal tuberculosis. Rev Soc Bars Med Trop: 33:253-257.

Nishimura T, Hasegawa N, Mori M, Takebayashi T, Harada N, Higuchi K, Tasaka S and Ishizaka A (2014). Accuracy of an interferon-gamma release assay to detect active pulmonary and extrapulmonary tuberculosis. Int J Tuberc Lung Dis; 12: 269-274. 
Ongut G, Ogunc D, Gunseren F, Ogus C, donnez L, Colak D and Gultekin M (2013). Evaluation of the ICT Tuberculosis test for the routine diagnosis of tuberculosis. BMC Infectious Diseases; 6: 37-41.

Rasolofo V. Rasolonavalona T, Ramarkota $\mathrm{H}$, Chanteau S (2011). Predictive values of ICT Tuberculosis test for the routine diagnosis of tuberculosis in Madagascar. Int J Tuberc Lung Dis; 4: 184-185.

Ravn P, Munk ME, Andersen AB, Lundgren B, Lundgren JD, Nielsen LN, Kok-Jensen A, Andersen P and Weldingh J (2010). Prospective evaluation of a whole-blood test using Mycobacterium tuberculosisspecific antigens ESAT-6 and CFP-10 for diagnosis of active tuberculosis. Clin Diagn Lab Immunol; 12: 491496.

Song K, Jeon JH, Park WB, Kim SH, Park KU, Kim NJ, Oh M, Kim HB and Choe KW (2011).

Usefulness of the whole blood interferon-gamma release assay for diagnosis of extrapulmonary tuberculosis. Diagnosis Microbiology and Infectious Disease:63: 182-187.

Steingart KR, Henry M, Laal S, Hopewell PC, Ramsay A, Menzies D, Cunningham J, weldingh K and Pai M (2013). Commercial serological antibody detection tests for the diagnosis of pulmonary tuberculosis: a systematic review. PLoS Med: 4: e202.

Takahashi T and Nakayama T (2011). Novel Technique of Quantitative Nested Real-Time PCR Assay for Mycobacterium tuberculosis DNA. Journal of clinical microbiology; 44 (3): 1029-1039.

Torrea G. Van de Perre P, Quedraogo M, Zougba A, Sawadogo A, Dingtoumda B, Diallo B, Defer MC, Sombie I, Zanetti S, Sechi LA (2014). PCR-based detection of the Mycobacterium tuberculosis complex in urine of HIV-infected and uninfected pulmonary and extrapulmonary tuberculosis patients in Burkina Faso. J Med Microbial; 54: 39-44.

Tsiouris SJ, Coetzee D, Toro PL, Austin J, Stein Z and El-Sadr W (2012). Sensitivity analysis and potential uses of a novel gamma interferon release assay for diagnosis of tuberculosis. J Clin Microbiol; 44: 2844-18 